\title{
Diet quality, change in diet quality and risk of incident CVD and diabetes
}

\author{
Zhe Xu' ${ }^{1}$, Lyn M Steffen ${ }^{2}$, Elizabeth Selvin ${ }^{1}$ and Casey M Rebholz ${ }^{1, *}$ \\ 'Department of Epidemiology, Johns Hopkins Bloomberg School of Public Health, Baltimore, MD, USA: ${ }^{2}$ Division of \\ Epidemiology and Community Health, University of Minnesota School of Public Health, Minneapolis, MN, USA
}

Submitted 25 September 2018: Final revision received 29 March 2019: Accepted 7 May 2019: First published online 12 September 2019

\begin{abstract}
Objective: The objective of this study was to assess the prospective association between diet quality, as well as a 6-year change in diet quality, and risk of incident CVD and diabetes in a community-based population.

Design: We used Cox regression models to estimate the prospective association between diet quality, assessed using the Healthy Eating Index (HEI)-2015 and the Alternative HEI (AHEI)-2010 scores, as well as change in diet quality, and incident CVD and diabetes.

Setting: The ARIC Study recruited 15792 black and white men and women (45-64 years) from four US communities.

Participants: We included 10808 study participants who reported usual dietary intake via FFQ at visit 1 (1987-1989) and who had not developed CVD, diabetes, or cancer at baseline.

Results: Overall, 3070 participants developed CVD (median follow-up of 26 years) and 3452 developed diabetes (median follow-up of 22 years) after visit 1 . Higher diet score at the initial visit was associated with a significantly lower risk of CVD (HR per $10 \%$ higher HEI-2015 diet quality score: 0.90 (95\% CI: 0.86, 0.95) and HR per $10 \%$ higher AHEI-2010 diet quality score: 0.96 (95\% CI: 0.93, 0.99)). We did not observe a significant association between initial diet score and incident diabetes. There were no significant associations between change in diet score and CVD or diabetes risk in the overall study population.

Conclusions: Higher diet quality assessed using HEI-2015 and AHEI-2010 was strongly associated with lower CVD risk but not diabetes risk within a middle-aged, community-based US population.
\end{abstract}

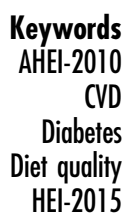

Keywords
AHEl-2010
CVD
Diabetes
Diet quality
HEI-2015
CVD remains the leading cause of death and a major cause of morbidity in the US and globally ${ }^{(1)}$. As a potent risk factor for CVD, diabetes mellitus is one of the fastest growing diseases $^{(2)}$. From 1988 to 2010 , the prevalence of diabetes has increased substantially and 21 million adults are affected in the USA ${ }^{(3)}$. Diet is an important modifiable risk factor for chronic diseases, including both CVD and diabetes mellitus ${ }^{(4)}$. Evidence from both observational studies and clinical trials has accumulated over the past two decades, highlighting the importance of individual nutrients, foods, and dietary patterns for CVD and diabetes prevention and management ${ }^{(5-9)}$.

The 2015-2020 Dietary Guidelines for Americans outline recommendations for dietary intake for the US population. As measures of diet quality, the Health Eating Index (HEI) assesses adherence to the dietary guidelines and the Alternative Healthy Eating Index (AHEI) assesses dietary components that have been associated with a lower risk of chronic diseases ${ }^{(9)}$. A series of studies conducted in large, US-based, multi-ethnic cohorts have demonstrated that higher levels of diet quality, assessed using the 2010 version of the HEI and the AHEI, were associated with a lower risk of diabetes, CVD, and mortality from CVD, cancer, or all causes combined ${ }^{(9-15)}$. HEI-2015 is the latest iteration of the index and was updated with the most recent (2015-2020) Dietary Guidelines ${ }^{(16,17)}$. A higher HEI-2015 score was related to lower mortality risk in a multi-ethnic population $^{(18)}$. However, the association between diet quality measured by HEI-2015 and the incidence of diabetes and CVD has not been evaluated.

Previous research has identified an improvement in dietary intake in the USA over the past few decades, with 
an increased consumption of whole grains, fruits and vegetables, but overall diet quality remains far from optimal $^{(19-21)}$. There is limited evidence on individual-level change in diet quality and subsequent health outcomes. In studies of predominantly Caucasian nurses and other health professionals in the USA, deterioration of diet quality was associated with an elevated risk of CVD and diabetes, and improvement in diet quality was associated with a lower risk of CVD and diabetes ${ }^{(22,23)}$. It is not known whether these findings can be extended to other segments of the US population.

The objective of the present study was to assess the prospective association between diet quality, as well as a 6-year change in diet quality, and subsequent risk for incident CVD and diabetes in a community-based population of men and women, both black and white.

\section{Methods}

\section{Study population and design}

The Atherosclerosis Risk in Communities (ARIC) Study is a community-based, prospective cohort study, which recruited 15792 participants aged 45 to 64 years at baseline from four US communities (Washington County, Maryland; Forsyth County, North Carolina; Jackson, Mississippi; and Minneapolis, Minnesota) ${ }^{(24)}$. The first study visit occurred in 1987 to 1989. Follow-up examination visits were conducted in 1990 to 1992 (visit 2), 1993 to 1995 (visit 3), 1996 to 1998 (visit 4), and 2011 to 2013 (visit 5). Annual follow-up telephone calls were also conducted from 1987.

In this prospective analysis of the ARIC Study, we examined the association between initial diet quality (at visit 1) as well as diet quality change from visit 1 to visit 3 and incident CVD and incident diabetes. Participants who developed diabetes, CVD or cancer at baseline were excluded ( $n$ 3738). We also excluded participants with implausible energy intake $(<2510 \mathrm{~kJ} \quad(<600 \mathrm{kcal})$ or $>17573 \mathrm{~kJ}$ $(>4200 \mathrm{kcal})$ per day for men and $<2092 \mathrm{~kJ}$ ( $<500 \mathrm{kcal})$ or $>15062 \mathrm{~kJ}$ ( $>3600 \mathrm{kcal})$ per day for women) ( $n$ 256), missing dietary data $(n 71)$, neither white nor African American participants from Minneapolis, Minnesota and Washington County, Maryland ( $n$ 80), missing covariates (age, sex, physical activity, smoking status, education, BMI, estimated glomerular filtration rate (eGFR) level, hypertension, and hypercholesterolaemia status) ( $n$ 642), and missing CVD or diabetes diagnosis ( $n$ 197). A total of 10808 participants were included in the analysis of diet quality (visit 1) and incident CVD and diabetes (Supplemental Figure 1A).

For the analysis of change in diet quality, incident CVD and incident diabetes, we excluded participants with prevalent diabetes, CVD, or cancer prior to visit 3 ( $n$ 4228) from the 12887 participants who attended visit 3.
We also excluded participants with implausible energy intake ( $n$ 403), missing dietary data ( $n$ 117), neither white nor African American, or African American participants from Minneapolis, Minnesota and Washington County, Maryland ( $n$ 51), missing covariates ( $n$ 509) measured at visit 3 , and missing CVD or diabetes diagnosis ( $n$ 152). A total of 7427 participants were included in the analysis of change in diet quality, incident CVD and incident diabetes (Supplemental Figure 1B).

\section{Dietary intake assessment}

Dietary intake was assessed at visit 1 and visit 3 using a validated semi-quantitative 66-item FFQ, which was modified from the Willett $\mathrm{FFQ}^{(25-27)}$. Participants were asked to report the average frequency of each food item they consumed of a particular portion size in the preceding year. Nutrient intake was calculated by multiplying self-reported frequency of consumption and portion size by the nutritional content of each food item using US Department of Agriculture data sources.

The Healthy Eating Index (HEI)-2015 and the Alternative Healthy Eating Index (AHEI)-2010 were used to represent diet quality and adherence to healthy eating for chronic disease prevention. HEI-2015 is the latest iteration of the index and was designed to assess adherence to the 2015-2020 Dietary Guidelines for Americans ${ }^{(16,17)}$. It consists of thirteen components that sum to a total maximum score of 100 points: total fruits, whole fruits, total vegetables, greens and beans, whole grains, dairy, total protein foods, seafood and plant proteins, fatty acids, refined grains, sodium, added sugars, and saturated fats. In the AHEI-2010, which was created to reflect foods and nutrients that have been consistently associated with a lower risk of chronic diseases, there are 11 food components that sum to a total maximum score of 110 : vegetables, fruits, whole grains, nuts and legumes, long-chain $n-3$ fatty acids, PUFA (excluding long-chain $n-3$ fatty acids), red/processed meat, sugar-sweetened beverages and fruit juice, trans fat, sodium, and alcohol $^{(9,23)}$.

Diet quality at visit 1 was classified using quintiles of HEI-2015 and AHEI-2010 scores. We categorized change in diet quality scores over a six-year period from visit 1 to visit 3 as follows: large decrease in the diet quality score $(<-10 \%)$, small to moderate decrease $(\geq-10 \%$ to $<-3 \%)$, no change or stable $(-3 \%$ to $+3 \%)$, small to moderate increase $(>+3 \%$ to $\leq+10 \%)$, and large increase $(>+10 \%)$. We also expressed change in diet quality using nine categories representing consistently low diet quality, consistently moderate diet quality, consistently high diet quality, and inconsistent diet quality scores (low to moderate; low to high; moderate to high; moderate to low; high to moderate; high to low). 


\section{Ascertainment of incident CVD}

We defined incident CVD as the first occurrence of any of the following: CHD (including hospitalized myocardial infarction, fatal CHD); definite or probable stroke; or died or hospitalized with an ICD-9 code for heart failure. Details of incident events have been previously described $^{(24,28)}$. Briefly, events were identified through cohort annual telephone follow-up, active community surveillance, and linkage with vital statistics databases (National Death Index) for fatal CVD events. The event date was defined as the date of first hospital discharge with CVD diagnosis or death due to CVD. Outcomes were ascertained from baseline, which was visit 1 for the initial diet quality exposure, and visit 3 for the change in diet quality exposure, through 31 December 2016. Incident heart failure, $\mathrm{CHD}$, and stroke were also investigated separately as secondary outcomes.

\section{Ascertainment of incident diabetes}

Incident diabetes in this study was ascertained from visit 1 for the initial diet quality exposure and from visit 3 for the change in diet quality exposure through 31 December 2016. Incident diabetes was defined as self-report of a physician diagnosis, current glucose lowering medication use as determined during ARIC visits and annual followup phone interviews, or elevated blood glucose levels (fasting glucose $\geq 2270 \mathrm{mmol} / \mathrm{l}(\geq 126 \mathrm{mg} / \mathrm{dl})$ or non-fasting glucose $\geq 3604 \mathrm{mmol} / \mathrm{l}(\geq 200 \mathrm{mg} / \mathrm{dl})$ ) measured using the hexokinase method during ARIC visits $^{(29,30)}$. For participants with self-reported newly diagnosed diabetes, the diagnosis date was assigned to the midpoint between the last visit without diabetes and the next visit with diabetes; for diabetes defined by blood glucose levels, the diagnosis date was estimated as the point when the serum glucose concentration crossed the diabetes cutoffs on a regression line of glucose concentrations by visit date ${ }^{(31)}$

\section{Measurement of covariates}

Information about age, sex, race-centre, education level, physical activity, smoking status, family (parental) history of diabetes, and family (parental) history of CHD or stroke was collected via structured questionnaires administered to participants by trained interviewers at study visits. Selfreport of physical activity type, duration, frequency, and intensity was assessed using the Baecke questionnaire and leisure-time (sport) physical activity was expressed as a score ranging from 1 to 5 , with 5 being the most $\operatorname{active}^{(32,33)}$.

BMI was calculated as weight (in kilograms) divided by height (in metres) squared, using measurements taken during the study visits. Blood pressure was measured via a standardized protocol, and the mean of the second and third of three readings was used. Hypertension was defined as systolic blood pressure $\geq 140 \mathrm{mmHg}$, diastolic blood pressure $\geq 90 \mathrm{mmHg}$, or use of blood pressure lowering medication within the past 2 weeks. Hypercholesterolaemia was defined as total cholesterol $\geq 4324 \mathrm{mmol} / \mathrm{l}(\geq 240 \mathrm{mg} / \mathrm{dl})$ or self-reported use of cholesterol lowering medication within the past 2 weeks ${ }^{(34)}$. eGFR was calculated using the Chronic Kidney Disease Epidemiology (CKD-EPI) 2009 equation which incorporates serum creatinine, age, sex and race $^{(35)}$.

\section{Statistical analysis}

We used descriptive statistics to report the overall HEI-2015 and AHEI-2010 diet quality scores as well as the scores and intake amounts of specific food and nutrient components of these diet quality scores at visit 1 and visit 3 . Differences in total diet scores and scores for individual food items between visit 1 and visit 3 were evaluated using paired $t$-tests. Study participant characteristics at visit 1 were reported across quintiles of diet quality at visit 1 . We also reported participant characteristics at visit 3 as well as the average change in intake for each component according to categories of change in diet quality. Differences in participant characteristics across quintiles of diet quality and categories of change in diet quality were tested using ANOVA for continuous variables and Pearson's chi-squared test for dichotomous and categorical variables.

Cox proportional hazard regression was used to estimate hazard ratios (HR) and 95\% CI for the association between initial (visit 1) diet quality (HEI-2015, AHEI-2010) and incident CVD and incident diabetes, incorporating time to event. We also used Cox proportional hazard regression to estimate the association between change in diet quality and incident CVD and diabetes. Time since baseline (which was visit 1 for analysis of initial diet quality and visit 3 for the analysis of change in diet quality) was used as the time metric. Person-years were calculated by using time from baseline visit date until an event, loss to follow-up, or 31 December 2016, whichever occurred first. Trend was tested using an ordinal variable for quintile of diet quality score in Cox regression. The proportional hazards assumption for Cox regression models was checked by using Schoenfeld residuals (estat phtest command in Stata), and no major violations were detected.

Different regression models were developed to account for potential confounders. For the analyses of initial diet score as the exposure, we developed these models. Model 1 was adjusted for age (continuous), sex, race-centre, education level (<high school, high school, > high school), family history of diabetes (yes/no), family history of CHD or stroke (yes/no), smoking status (current/former/never smoker), physical activity (quintile), and total energy intake (quintile). We used a combined term for race and centre given the non-uniform distribution of racial groups across centres in the ARIC study. Model 2 was additionally adjusted for hypertension status (yes/no), hypercholesterolaemia status (yes/no), and eGFR (quintile). In Model 3, we adjusted 
for all the covariates in Model 2 plus BMI category $(<25$, $\geq 25-<30, \geq 30 \mathrm{~kg} / \mathrm{m}^{2}$ ). In addition, levels in alcohol intake were included as a covariate in models for HEI-2015, but not for AHEI-2010, since alcohol intake was a component of the AHEI-2010 score.

For the analyses of change in diet quality as the exposure, we adjusted for the same covariates measured at visit 3 (visit 1 eGFR level was used because serum creatinine was not measured at visit 3), and additionally adjusted for the initial diet quality score (quintile), change in smoking status (categorical), change in physical activity (quintile), change in total energy intake (quintile), and change in BMI (quintile).

In order to separate the risk associated with the initial diet score level from change in diet quality, we conducted two analyses: (i) an analysis of diet trajectories in which we categorized both visit 1 and visit 3 diet quality scores as low, moderate, or high using tertiles, and (ii) a stratified analysis by category of initial (visit 1) diet quality score categorized as low, moderate, or high using tertiles.

To assess the robustness of our findings, we conducted subgroup analyses according to sex, race, education level and BMI category. Effect modification by these factors was assessed by conducting tests of interaction.

All analyses were conducted using the statistical software package Stata version 14. Statistical significance was assessed using a two-sided probability of alpha-level of 0.05 .

\section{Results}

\section{Baseline characteristics according to initial diet quality (visit 1)}

Among the 10808 participants in the analytic study population, the mean age at baseline was 53.7 (SD 5.7) years, $23.3 \%$ were black, and $56.1 \%$ were women. Participants with higher HEI-2015 diet quality score were more likely to be older, female, white, more highly educated, not current smokers, had lower alcohol intake, had a higher level of physical activity, and were more likely to have hypercholesterolaemia (Table 1). Mean baseline weight and BMI were lower among those who had a higher diet quality score. Similar patterns were observed across quintiles of AHEI-2010 score.

There was a substantial difference in intake of each component across quintiles of initial diet quality score for HEI-2015 (Supplemental Table 1) and AHEI-2010 (Supplemental Table 2). For example, dietary intake of total fruit was higher in the highest $v$. lowest quintile of HEI-2015 (quintile 5: 0.54 cup equivalents/1000 kJ $v$. quintile 1: $0 \cdot 18$ cup equivalents/1000 kJ (quintile 5: $2 \cdot 28$ cup equivalents $/ 1000 \mathrm{kcal} v$. quintile 1: 0.75 cup equivalents $/ 1000 \mathrm{kcal})$ ). Similar patterns were observed for AHEI-2010, e.g. dietary intake of fruit was 2.15 servings/d in quintile $5 v \cdot 0 \cdot 80$ servings/d in quintile 1.

\section{Change in diet quality}

There was a small, but statistically significant increase in mean diet quality scores over the six-year period for HEI-2015 (visit 1: 71.0 (SD 8.7), visit 3: 72.9 (SD 8.4); Supplemental Table 3) and AHEI-2010 (visit 1: 50.7 (SD 12.1), visit 3: $52 \cdot 4$ (SD 11.3); Supplemental Table 4) (both $P<0.001$ ). Scores for the consumption of fruit and vegetables were significantly higher at visit 3 relative to visit 1. For HEI-2015, participants had significant improvements in diet scores for greens and beans, fatty acids, added sugars, and saturated fats, and a decrease in diet component scores for total protein foods and seafood/plant proteins. For AHEI-2010, diet component scores increased over time for red/processed meat, trans fat, and long-chain ( $n-3)$ fats, and scores decreased over time for PUFA, sodium and alcohol. Those participants with a large $(>10 \%)$ increase in HEI-2015 score reduced added sugars by $1.5 \%$ of energy and reduced saturated fat by $3.0 \%$ of energy (Supplemental Table 5 ). Those participants with a large increase in AHEI-2010 score increased their intake of $n-3$ fatty acids by $58.1 \mathrm{mg} / \mathrm{d}$ and increased their intake of sodium by $24.4 \mathrm{mg} / \mathrm{d}$ on average (Supplemental Table 6).

\section{Baseline characteristics (visit 3) according to change in diet quality}

Participants with an increase in HEI-2015 diet quality scores had lower diet quality scores at visit 1 , were more likely to be younger, male, and black (Supplemental Table 7). Mean weight and BMI at visit 3 were the lowest for those with stable or small-to-moderate increase in diet quality. On average, weight and BMI increased over the six-year period, but participants with a large increase in diet quality tended to have less weight gain. Similar patterns, although less noticeable differences, were observed across the five categories of change in AHEI-2010 score.

Initial diet quality and incident CVD and diabetes A total of 3080 participants developed CVD and 3452 developed incident diabetes after visit 1 during a median follow-up of 26 years and 22 years, respectively. The overall crude CVD incidence rate was 13.1 per 1000 person-years (95\% CI: 12.6, 13.6 per 1000 person-years). The crude incidence rate for diabetes was 16.9 per 1000 person-years (95\% CI: 16.4, 17.5 per 1000 person-years).

Higher initial diet quality score was significantly associated with a $16 \%$ to $21 \%$ reduced risk of incident CVD (HEI-2015: HR for quintile 5 v. 1, 0.79; $95 \%$ CI: 0.70 , 0.91; AHEI-2010: HR for quintile 5 v. 1, 0.84; $95 \% \mathrm{CI}$ : $0.74,0.95)$ in Model 3, which adjusted for age, sex, race-centre, education, family history of diabetes, family history of $\mathrm{CHD} /$ stroke, smoking status, physical activity, alcohol intake (only for HEI-2015), total energy intake, hypertension status, hypercholesterolaemia status, eGFR, and BMI category (Table 2). There was a significant inverse 
Table 1 Baseline (visit 1, 1987-1989) characteristics of participants by quintiles of initial diet quality score

\begin{tabular}{|c|c|c|c|c|c|c|c|c|c|c|c|c|c|c|}
\hline \multirow[b]{3}{*}{ Characteristic } & \multicolumn{7}{|c|}{ HEI-2015 } & \multicolumn{7}{|c|}{ AHEI-2010 } \\
\hline & \multicolumn{2}{|c|}{ Quintile 1} & \multicolumn{2}{|c|}{ Quintile 3} & \multicolumn{2}{|c|}{ Quintile 5} & \multirow[b]{2}{*}{$P$ value } & \multicolumn{2}{|c|}{ Quintile 1} & \multicolumn{2}{|c|}{ Quintile 3} & \multicolumn{2}{|c|}{ Quintile 5} & \multirow[b]{2}{*}{$P$ value } \\
\hline & $n$ or mean & $\%$ or SD & $n$ or mean & $\%$ or SD & $n$ or mean & $\%$ or SD & & $n$ or mean & $\%$ or SD & $n$ or mean & $\%$ or SD & $n$ or mean & $\%$ or SD & \\
\hline Participants, $n$ & 2162 & & 2161 & & 2161 & & & 2162 & & 2161 & & 2161 & & \\
\hline Diet score & 58.2 & 4.4 & $70 \cdot 7$ & 1.2 & 82.5 & 3.6 & $<0.001$ & 62.7 & $7 \cdot 0$ & 70.5 & $6 \cdot 8$ & 78.4 & $6 \cdot 8$ & $<0.001$ \\
\hline Energy intake $(\mathrm{kJ} / \mathrm{d})$ & 7385 & 2837 & 6920 & 2473 & 6096 & 2071 & $<0.001$ & 7146 & 2623 & 6657 & 2594 & 6878 & 2301 & $<0.001$ \\
\hline Age (years) & 53.1 & 5.6 & 53.7 & $5 \cdot 7$ & 54.5 & $5 \cdot 7$ & $<0.001$ & 53.0 & $5 \cdot 6$ & 53.7 & 5.7 & $54 \cdot 3$ & $5 \cdot 7$ & $<0.001$ \\
\hline Female & 889 & $41 \cdot 1$ & 1250 & $57 \cdot 8$ & 1498 & $69 \cdot 3$ & $<0.001$ & 961 & 44.4 & 1206 & $55 \cdot 8$ & 1416 & $65 \cdot 5$ & $<0.001$ \\
\hline Black & 520 & $24 \cdot 1$ & 597 & $27 \cdot 6$ & 378 & 17.5 & $<0.001$ & 573 & $26 \cdot 5$ & 555 & $25 \cdot 7$ & 328 & $15 \cdot 2$ & $<0.001$ \\
\hline \multicolumn{15}{|l|}{ Education level } \\
\hline$<$ high school & 644 & 29.8 & 438 & $20 \cdot 3$ & 257 & 11.9 & $<0.001$ & 546 & $25 \cdot 3$ & 456 & $21 \cdot 1$ & 268 & $12 \cdot 4$ & $<0.001$ \\
\hline$\geq$ high school & 1518 & $70 \cdot 2$ & 1723 & $79 \cdot 7$ & 1904 & $88 \cdot 1$ & & 1616 & 74.7 & 1705 & 78.9 & 1893 & $87 \cdot 6$ & \\
\hline \multicolumn{15}{|l|}{ Smoking status } \\
\hline Current smoker & 802 & $37 \cdot 1$ & 519 & $24 \cdot 0$ & 345 & $16 \cdot 0$ & $<0.001$ & 667 & 30.9 & 566 & $26 \cdot 2$ & 424 & $19 \cdot 6$ & $<0.001$ \\
\hline Former smoker & 668 & 30.9 & 625 & 28.9 & 763 & $35 \cdot 3$ & & 622 & 28.8 & 648 & $30 \cdot 0$ & 811 & 37.5 & \\
\hline Never smoker & 692 & 32.0 & 1017 & $47 \cdot 1$ & 1053 & $48 \cdot 7$ & & 873 & 40.4 & 947 & $43 \cdot .8$ & 926 & $42 \cdot 9$ & \\
\hline Physical activity index & $2 \cdot 3$ & 0.7 & 2.4 & 0.8 & $2 \cdot 7$ & 0.8 & $<0.001$ & $2 \cdot 3$ & 0.8 & 2.5 & 0.8 & 2.7 & 0.8 & $<0.001$ \\
\hline Alcohol intake (drinks/d) & 0.6 & 1.2 & 0.4 & 1.0 & 0.3 & 0.6 & $<0.001$ & 0.5 & 1.3 & 0.5 & 0.9 & 0.5 & 0.8 & 0.002 \\
\hline Weight (lb) & $173 \cdot 6$ & $36 \cdot 2$ & $170 \cdot 4$ & 35.5 & $162 \cdot 8$ & 33.5 & $<0.001$ & 174.6 & $36 \cdot 1$ & $170 \cdot 3$ & $35 \cdot 7$ & $165 \cdot 5$ & 34.3 & $<0.001$ \\
\hline $\mathrm{BMI}\left(\mathrm{kg} / \mathrm{m}^{2}\right)$ & $27 \cdot 1$ & 5.0 & $27 \cdot 3$ & $5 \cdot 0$ & $26 \cdot 6$ & 4.9 & $<0.001$ & 27.4 & $5 \cdot 2$ & $27 \cdot 2$ & $5 \cdot 1$ & $26 \cdot 8$ & 4.8 & $<0.001$ \\
\hline \multicolumn{15}{|l|}{ BMI level } \\
\hline$<25 \mathrm{~kg} / \mathrm{m}^{2}$ & 792 & $36 \cdot 6$ & 729 & 33.7 & 943 & $43 \cdot 6$ & $<0.001$ & 758 & 35.1 & 783 & $36 \cdot 2$ & 821 & $38 \cdot 0$ & 0.007 \\
\hline$\geq 25-<30 \mathrm{~kg} / \mathrm{m}^{2}$ & 829 & $38 \cdot 3$ & 910 & $42 \cdot 1$ & 790 & $36 \cdot 6$ & & 870 & $40 \cdot 2$ & 862 & 39.9 & 894 & 41.4 & \\
\hline$\geq 30 \mathrm{~kg} / \mathrm{m}^{2}$ & 541 & 25.0 & 522 & $24 \cdot 2$ & 428 & $19 \cdot 8$ & & 534 & 24.7 & 516 & 23.9 & 446 & $20 \cdot 6$ & \\
\hline Hypertension & 610 & 28.2 & 699 & $32 \cdot 3$ & 649 & $30 \cdot 0$ & 0.01 & 651 & 30.1 & 682 & 31.6 & 607 & $28 \cdot 1$ & 0.02 \\
\hline Hypercholesterolaemia & 500 & 23.1 & 525 & $24 \cdot 3$ & 581 & $26 \cdot 9$ & 0.02 & 504 & $23 \cdot 3$ & 551 & 25.5 & 588 & $27 \cdot 2$ & $<0.001$ \\
\hline eGFR $\left(\mathrm{ml} / \mathrm{min}\right.$ per $\left.1.73 \mathrm{~m}^{2}\right)$ & $103 \cdot 0$ & 14.6 & $103 \cdot 3$ & 14.9 & $102 \cdot 1$ & $13 \cdot 3$ & 0.02 & $103 \cdot 2$ & $15 \cdot 0$ & 103.3 & $15 \cdot 0$ & $101 \cdot 6$ & $13 \cdot 2$ & $<0.001$ \\
\hline Family history of diabetes & 488 & $25 \cdot 4$ & 467 & 23.9 & 465 & $23 \cdot 2$ & 0.42 & 470 & $24 \cdot 1$ & 507 & $25 \cdot 6$ & 464 & $23 \cdot 2$ & 0.52 \\
\hline Family history of $\mathrm{CHD} /$ stroke & 1192 & 61.9 & 1209 & 62.4 & 1254 & $63 \cdot 1$ & $0 \cdot 10$ & 1177 & 60.9 & 1194 & 60.7 & 1244 & $63 \cdot 2$ & 0.32 \\
\hline
\end{tabular}

Continuous variables are presented as mean and SD, categorical variables as $n$ and \%. 


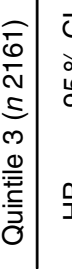

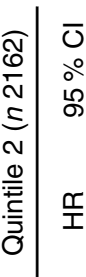

숭용용 ○ं 00 कే

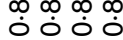
ㅇํㅇ융요 ó ó 형ㅎㅇㅇㅎํ vंọं

\section{ழํํ유}

둥ํㅇ

๓

守

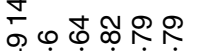

तें0ं0ं

กิ

ํㅛ요용

씽잉영

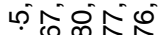

ㄷㅇㅇㅇㅇ

品

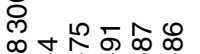

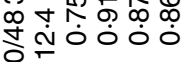

ठํ

뇽ㅇㅇ

ث்

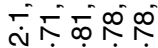

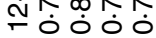

ธั

क

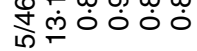

$\frac{1}{6}$

\section{பீ}

एก

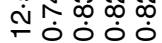

\&

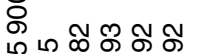

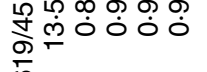

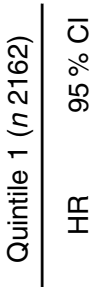

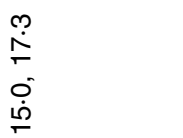

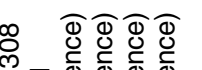

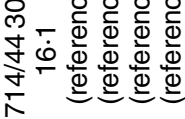

下

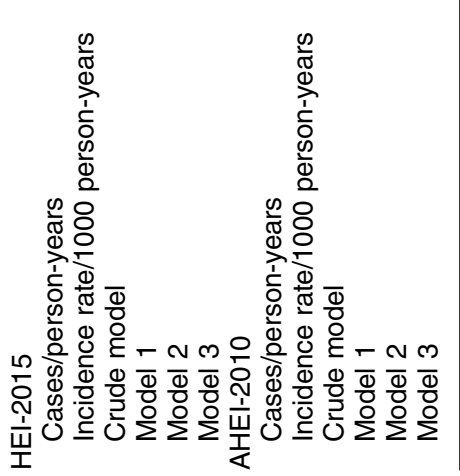

-

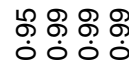

मल लं

0ं0

ิํㅇ

ó ó

ธํํㅇํํㅇㅇํㅇ융

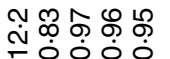

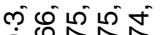

응ㅇㅇㅇㅇㅇ

○

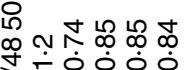

商

영 $88 \%$

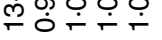

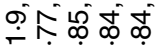

웅

+

응

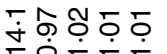

บ

넝ㅇํㅇ

$\because$

웅ㅎㅎ 항

ô

我号莗号

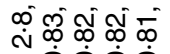

$\infty$

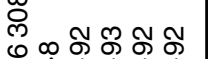

宓 ஹं。்

เุ

กั

ஸ்

㐫 Ðळळ

กิ的市

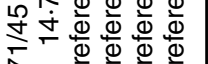

trend in CVD risk across quintiles of HEI-2015 diet quality score $(P$ trend $<0 \cdot 001)$ and across quintiles of AHEI-2010 diet quality score $(P$-trend $=0.03)$. There was a $10 \%$ lower risk of CVD per $10 \%$ higher HEI-2015 score (HR, 0.90; $95 \%$ CI: $0 \cdot 86,0.95)$ and a $4 \%$ lower risk of CVD per $10 \%$ higher AHEI-2010 score (HR, 0.96; $95 \%$ CI: 0.93, 0.99).

These findings for HEI-2015 and CVD were generally consistent with the results for AHEI-2010 and specific types of CVD (incident heart failure, CHD, and stroke), and were most pronounced for stroke (HR, 0.65; $95 \%$ CI: 0.51, 0.84; Fig. 1). For AHEI-2010, there was a significant trend for increasing quintiles and lower risk of heart failure (HR, 0.83, $95 \%$ CI: $0.70,0.97 ; P$ trend $=0.04$ ), but not for CHD or stroke. We did not observe a significant association between initial diet quality score (both HEI-2015 and AHEI-2010) and incident diabetes.

\section{Diet quality change and incident CVD and diabetes}

We documented 1794 CVD cases and 1984 diabetes cases after visit 3 during a median follow-up of 20 years and 18 years, respectively. The crude CVD incidence rate after visit 3 was 13.7 per 1000 person-years (95\% CI: 13.1, 14.3 per 1000 person-years). The crude incidence rate after visit 3 of diabetes was 17.2 per 1000 person-years (95\% CI: 16.4, $17 \cdot 9$ per 1000 person-years).

There were no statistically significant associations between change in diet score and CVD risk based on HEI-2015, except for a slightly higher risk among those with a large increase in AHEI-2010 compared with the stable group by AHEI-2010 (HR, 1.22; $95 \%$ CI: 1.02, 1.47) (Supplemental Table 8 ). There was also no statistically significant association between change in diet quality and diabetes risk in the fully adjusted model (Supplemental Table 9). We observed an association between a large decrease in AHEI-2010 score and incident diabetes after adjusting for demographic characteristics, socio-economic factors, family history of disease, lifestyle factors, and initial diet quality score in Model 1 (HR, 1.22; $95 \%$ CI: 1.02, 1.46), but the association was attenuated and no longer statistically significant after accounting for change in other health behaviours in Model 2 and further adjustment for confounders in Models 3 and 4.

Compared with the participants with consistently high HEI-2015 scores over the six-year change period, participants who decreased from the highest to the lowest tertile had a $40 \%$ higher risk of incident diabetes (HR, 1.40; $95 \%$ CI: $1.01,1.96)$ in the fully adjusted model (Supplemental Tables 10). Additionally, there was a significant increased risk of diabetes for those participants who had a decrease in diet quality from the highest to the moderate tertile for AHEI-2010 over the six-year change period compared with those with consistently high diet quality (HR, 1.22; $95 \% \mathrm{CI}$ : 1.01, 1.48). No significant associations were observed for 

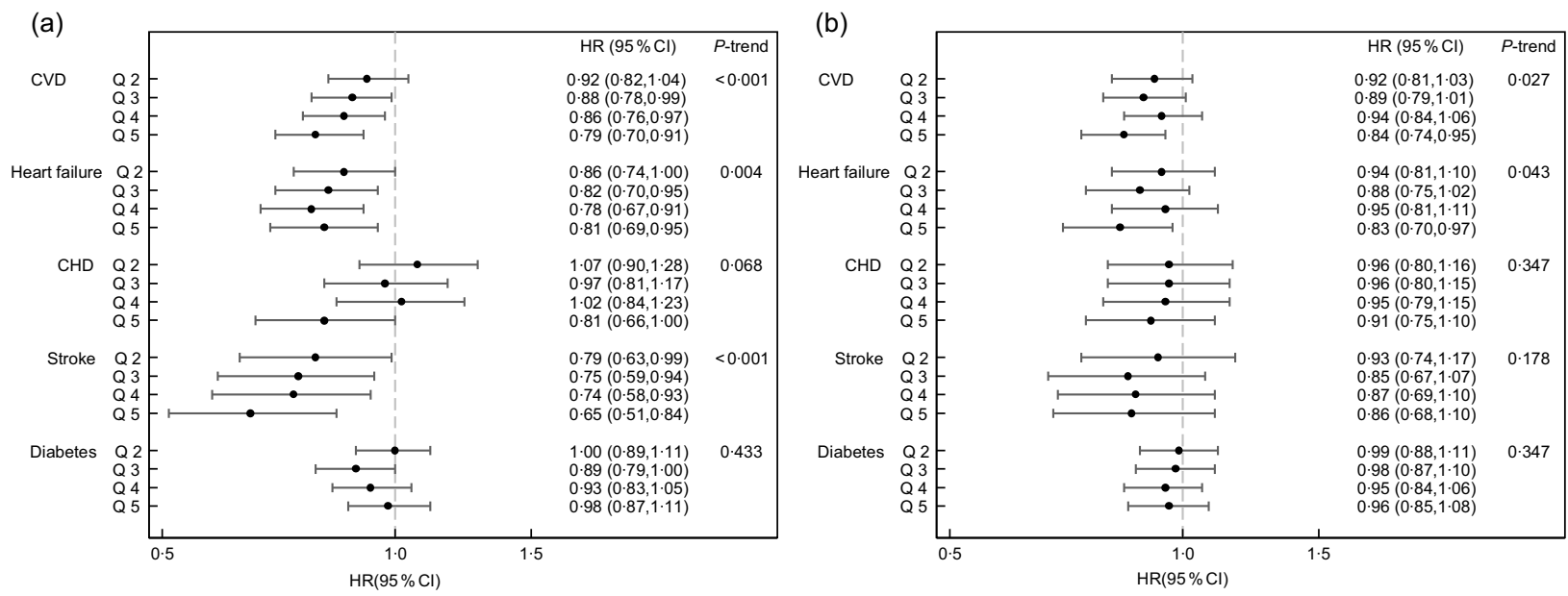

Fig. 1 Risk* of incident CVD and incident diabetes according to quintile of initial diet quality score for HEI-2015 (a) and AHEI-2010 (b). *Hazard ratios calculated using quintile 1 as reference group, adjusted for age, sex, race-centre, education, family history of diabetes, family history of $\mathrm{CHD} /$ stroke, smoking status, physical activity, alcohol intake (only for HEI), total energy intake, hypertension status, hypercholesterolaemia status, eGFR, and BMI category (Model 3)

the nine categories of change in diet quality (for both HEI-2015 and AHEI-2010) and CVD risk.

For the analysis stratified by initial diet quality score, there was no significant associations for categories of change in diet quality and risk of CVD (Supplemental Table 11). Among those with a high initial HEI-2015 score, a large increase in diet quality $(>10 \%)$ was significantly associated with a lower risk of diabetes (Model $4 \mathrm{HR}$, 0.44; $95 \%$ CI: 0.26, 0.78) (Supplemental Tables 12).

\section{Subgroup analyses}

The inverse association between diet quality score at visit 1 and incident CVD was consistent across subgroups by sex, race, education level, and BMI categories using HEI-2015 and AHEI-2010 (all $P$ values for interaction $>0.05$ ) (Supplemental Figure 2).

\section{Discussion}

In the present study, higher initial diet score was associated with a significantly lower risk of incident CVD, but not significantly associated with incident diabetes within a community-based population of middle-aged men and women, black and white. This relationship was consistent across population subgroups, including sex, race, education level and BMI category. Diet quality slightly improved over a six-year period between the late 1980s and the 1990s, but there was no consistent and significant association between change in diet quality and risk of incident CVD or incident diabetes in the overall study population.

Our findings are partially consistent with previous research. In line with previous epidemiologic studies using the earlier version of HEI and AHEI-2010 diet scores, we observed a strong association between the higher diet quality and reduced risk of CVD using both the HEI-2015 and AHEI-2010 ${ }^{(5-9)}$. Although HEI and AHEI diet scores were developed for slightly different purposes, they both capture essential elements of a high quality $\operatorname{diet}^{(22)}$, including healthy foods, such as fruits, vegetables, whole grains and unsaturated fats, as well as potentially detrimental foods and nutrients, such as sodium. There was a stronger and more consistent dose-response relationship between HEI-2015 and CVD risk than AHEI-2010, especially for the secondary outcomes of heart failure and stroke. A possible explanation might be the fact that HEI-2015 and AHEI-2010 vary with respect to their components and optimal cut-offs. Compared with AHEI-2010, HEI-2015 has a separate component for protein from seafood and plant sources. Previous research has shown that seafood and plant protein was significantly associated with a lower risk of CVD compared with other food sources of protein $^{(36)}$. In AHEI-2010, there was no separate component for seafood. The stronger association between HEI-2015 and CVD might be driven by separately accounting for different food sources of protein.

Previous analyses conducted in the Nurses' Health Study (NHS) and the Health Professionals Follow-up Study (HPFS) have demonstrated that HEI-2005 and AHEI-2010 were strongly associated with diabetes risk ${ }^{(9)}$. However, we did not observe such an association between HEI-2015 and AHEI-2010 score and diabetes risk in the present analysis of the ARIC Study population. Our findings are consistent with a previous analysis of HEI-2010 and diabetes risk conducted among participants in the Multi-ethnic Cohort $^{(10)}$. As such, diet quality, as assessed using HEI-2015 and AHEI-2010, may have limited benefit for preventing diabetes in more diverse, general populations. 
Few studies have previously investigated the association between change in HEI-2015 score and CVD or diabetes risk. Deterioration in diet quality as assessed using AHEI-2010 was associated with higher risk of CVD and diabetes in the NHS and the HPFS populations ${ }^{(22,23)}$. However, we were not able to replicate these findings for AHEI-2010 in the present study, nor were we able to demonstrate an association with change in HEI-2015 and cardiometabolic outcomes. The inconsistency in these findings might be due to differences in these studies with respect to the assessment of dietary intake, study populations, and incidence rates. Information on dietary intake was collected with a 66-item FFQ in ARIC, while a 131-item FFQ was used in NHS and HPFS. The more detailed FFQ might be better suited to detect diet quality change than our study. In contrast with the participants in the NHS and HPFS who are relatively homogenous with respect to demographic and socioeconomic status (mostly white and well-educated health professionals), ARIC Study participants were enrolled from four distinct US regions representing both blacks and whites, both men and women, and a relatively wide range of socioeconomic status as indicated by education level. The mean age of ARIC population was also nearly 4 years older than the age of participants in NHS and HPFS. The overall incidence rate of CVD in the NHS and HPFS pooled study population was around 9 per 1000 person-years, which was lower than 13 per 1000 person-years in the present study. The incidence rate of diabetes was also much lower in the NHS and HPFS pooled study population than in our study $(5 v .17$ per 1000 person-years, respectively). Lifestyle factors, including diet quality and other important health behaviours, as well as the general health status among the health professionals were better than among ARIC Study participants. In our multivariable regression models, we additionally adjusted for eGFR as a measure of kidney function, which is an important predictor for $\mathrm{CVD}^{(37)}$, and this confounder was not accounted for in the NHS and HPFS studies.

Despite the lack of a significant association between change in diet quality and CVD or diabetes in the present study, recommending improvement in overall diet quality is still important. Although the quality of dietary intake has improved over the recent past in the US, it remains far from optimal ${ }^{(12)}$. We observed small changes in diet quality in this observational setting in the late 1980s and early 1990s, suggesting that population-level strategies that occurred during this time were not very effective for modifying dietary intake. Community-, household-, or individual-level interventions may be necessary to achieve meaningful change in diet quality. Previous research has demonstrated that comprehensive lifestyle interventions, incorporating physical activity and weight loss along with improving diet quality, improve blood pressure levels and could reduce the risk of chronic diseases ${ }^{(38)}$.

Our study has several strengths. We conducted the present study in a community-based population of black and white adults in the US, providing broader generalizability relative to previous research on this topic. To provide a comprehensive analysis of diet quality and cardiometabolic diseases, we included several important outcomes including overall CVD, diabetes, and secondary outcome of heart failure, coronary heart disease, and stroke. Owing to repeated assessment of dietary intake, we were able to quantify the change in diet quality whereas prior studies have predominantly used a single measure of diet quality. We used two measures of diet quality, including the latest version of the HEI to assess adherence to the Dietary Guidelines for Americans as well as the AHEI-2010, which assesses relevant aspects of the diet for chronic disease prevention. The study participants in our sample were followed for an extended period of time to allow for the ascertainment of many incident CVD and diabetes cases.

The limitations of this study also deserve consideration. Self-reported dietary intake by food frequency questionnaire is prone to recall bias and other types of error ${ }^{(36)}$. In addition, the 66-item FFQ may not have captured the whole diet. Another limitation is that the sample size in some sub-groups was small, which reduces the statistical power to detect significant associations. Finally, although we adjusted for many potential confounders and accounted for change in the covariates, we are not able to rule out the influence of residual and unmeasured confounding in this observational study.

In summary, we observed strong associations between higher diet quality, assessed using HEI-2015 and AHEI2010, with lower CVD risk in a community-based population of middle-aged black and white, men and women. There was a slight improvement in diet quality over a six-year period between the late 1980s and the 1990s. We did not observe significant and consistent associations between change in diet quality and CVD or diabetes risk. Our results provide evidence that adherence to dietary guidelines confer benefits for CVD prevention within the general US population. Further research might focus on determining the possible influence of longer-term change in diet quality on cardiometabolic diseases in large study populations.

\section{Acknowledgements}

Acknowledgements: The authors thank the staff and participants of the Atherosclerosis Risk in Communities (ARIC) Study for their important contributions. Financial support: The Atherosclerosis Risk in Communities study has been funded in whole or in part with federal funds from the National Heart, Lung, and Blood Institute, National Institutes of Health, Department of Health and Human Services (HHSN268201700001I, HHSN268201700002I, HHSN268201700003I, HHSN268201700004I, HHSN268 201700005I). C.M.R. was supported by a Mentored Research Scientist Development Award from the National Institute 
of Diabetes and Digestive and Kidney Disease (K01 DK107782). E.S. was supported by NIH/NIDDK grants K24DK106414 and R01DK089174. Z.X. was supported by the China Scholarship Council (CSC). The funders had no role in the design, analysis, or writing of this article. Conflicts of interest: None. Authorship: The authors' contributions to the manuscript were as follows: Z.X., C.M.R., and E.S. designed the research; E.S. and C.M.R. provided databases; Z.X. performed the statistical analysis; Z.X. drafted the manuscript; and L.M.S., E.S., and C.M.R. provided critical feedback on the manuscript. Z.X. and C.M.R. had primary responsibility for final content. All authors have read and approved the final manuscript. Ethics of human subject participation: This study was conducted according to the guidelines laid down in the Declaration of Helsinki and all procedures involving human subjects were approved by Institutional Review Boards at all participating study centres. Written informed consent was obtained from all subjects.

\section{Supplementary material}

To view supplementary material for this article, please visit https://doi.org/10.1017/S136898001900212X

\section{References}

1. Benjamin EJ, Virani SS, Callaway CW et al. (2018) Heart disease and stroke statistics-2018 update: a report from the American Heart Association. Circulation 137, e67-e492.

2. Khazrai YM, Defeudis G \& Pozzilli P (2014) Effect of diet on type 2 diabetes mellitus: a review. Diabetes Metab Res Rev $\mathbf{3 0}$, Suppl. 1, 24-33.

3. Selvin E, Parrinello CM, Sacks DB et al. (2014) Trends in prevalence and control of diabetes in the United States, 1988-1994 and 1999-2010. Ann Intern Med 160, 517-525.

4. Bhupathiraju SN \& Hu FB (2016) Epidemiology of obesity and diabetes and their cardiovascular complications. Circ Res 118, 1723-1735.

5. InterAct Consortium, Romaguera $\mathrm{D}$, Guevara $\mathrm{M}$ et al. (2011) Mediterranean diet and type 2 diabetes risk in the European Prospective Investigation into Cancer and Nutrition (EPIC) study: the InterAct project. Diabetes Care 34, 1913-1918.

6. Ley SH, Hamdy O, Mohan V et al. (2014) Prevention and management of type 2 diabetes: dietary components and nutritional strategies. Lancet Lond Engl 383, 1999-2007.

7. Esposito K, Maiorino MI, Ceriello A et al. (2010) Prevention and control of type 2 diabetes by Mediterranean diet: a systematic review. Diabetes Res Clin Pract 89, 97-102.

8. Liese AD, Nichols M, Sun X et al. (2009) Adherence to the DASH Diet is inversely associated with incidence of type 2 diabetes: the insulin resistance atherosclerosis study. Diabetes Care 32, 1434-1436.

9. Chiuve SE, Fung TT, Rimm EB et al. (2012) Alternative dietary indices both strongly predict risk of chronic disease. J Nutr 142, 1009-1018.

10. Jacobs S, Harmon BE, Boushey CJ et al. (2015) A prioridefined diet quality indexes and risk of type 2 diabetes: the Multiethnic Cohort. Diabetologia 58, 98-112.
11. Popkin BM, Zizza C \& Siega-Riz AM (2003) Who is leading the change?. U.S. dietary quality comparison between 1965 and 1996. Am J Prev Med 25, 1-8.

12. Wang DD, Leung CW, Li Y et al. (2014) Trends in dietary quality among adults in the United States, 1999 through 2010. JAMA Intern Med 174, 1587-1595.

13. Rehm CD, Peñalvo JL, Afshin A et al. (2016) Dietary intake among US adults, 1999-2012. JAMA 315, 2542-2553.

14. McCullough ML (2014) Diet patterns and mortality: common threads and consistent results. J Nutr 144, 795-796.

15. Liese AD, Krebs-Smith SM, Subar AF et al. (2015) The dietary patterns methods project: synthesis of findings across cohorts and relevance to dietary guidance. J Nutr 145, 393-402.

16. Krebs-Smith SM, Pannucci TE, Subar AF et al. (2018) Update of the healthy eating index: HEI-2015. J Acad Nutr Diet 118, 1591-1602.

17. Reedy J, Lerman JL, Krebs-Smith SM et al. (2018) Evaluation of the healthy eating index-2015. J Acad Nutr Diet 118, 1622-1633.

18. Panizza CE, Shvetsov YB, Harmon BE et al. (2018) Testing the predictive validity of the healthy eating index-2015 in the multiethnic cohort: is the score associated with a reduced risk of all-cause and cause-specific mortality? Nutrients 10, 452.

19. Popkin BM, Zizza C \& Siega-Riz AM (2003) Who is leading the change?. U.S. dietary quality comparison between 1965 and 1996. Am J Prev Med 25, 1-8.

20. Wang DD, Leung CW, Li Y et al. (2014) Trends in dietary quality among adults in the United States, 1999 through 2010. JAMA Intern Med 174, 1587-1595.

21. Rehm CD, JL Peñalvo, Afshin A et al. (2016) Dietary intake among US adults, 1999-2012. JAMA 315, 2542-2553.

22. Sotos-Prieto M, Bhupathiraju SN, Mattei J et al. (2015) Changes in diet quality scores and risk of cardiovascular disease among US men and women. Circulation 132, 2212-2219.

23. Ley SH, Pan A, Li Y et al. (2016) Changes in overall diet quality and subsequent type 2 diabetes risk: three U.S. prospective cohorts. Diabetes Care 39, 2011-2018.

24. The ARIC Investigators (1989) The atherosclerosis risk in communities (ARIC) study: design and objectives. Am J Epidemiol 129, 687-702.

25. Stevens J, Metcalf PA, Dennis BH et al. (1996) Reliability of a food frequency questionnaire by ethnicity, gender, age and education. Nutr Res 16, 735-745.

26. Willett WC, Sampson L, Stampfer MJ et al. (1985) Reproducibility and validity of a semiquantitative food frequency questionnaire. Am J Epidemiol 122, 51-65.

27. Shimakawa T, Sorlie P, Carpenter MA et al. (1994) Dietary intake patterns and sociodemographic factors in the atherosclerosis risk in communities study. ARIC study investigators. Prev Med 23, 769-780.

28. White AD, Folsom AR, Chambless LE et al. (1996) Community surveillance of coronary heart disease in the Atherosclerosis Risk in Communities (ARIC) study: methods and initial two years' experience. J Clin Epidemiol 49, 223-233.

29. Duncan BB, Schmidt MI, Pankow JS et al. (2003) Low-grade systemic inflammation and the development of type 2 diabetes: the atherosclerosis risk in communities study. Diabetes 52, 1799-1805.

30. Wei GS, Coady SA, Reis JP et al. (2015) Duration and degree of weight gain and incident diabetes in younger versus middle-aged black and white adults: ARIC, CARDIA, and the Framingham Heart Study. Diabetes Care 38, 2042-2049.

31. Wang L, Folsom AR, Zheng Z-J et al. (2003) Plasma fatty acid composition and incidence of diabetes in middle-aged adults: the Atherosclerosis Risk in Communities (ARIC) Study. Am J Clin Nutr 78, 91-98.

32. Baecke JA, Burema J \& Frijters JE (1982) A short questionnaire for the measurement of habitual physical activity in epidemiological studies. Am J Clin Nutr 36, 936-942. 
33. Cobb LK, Godino JG, Selvin E et al. (2016) Spousal influence on physical activity in middle-aged and older adults: the ARIC study. Am J Epidemiol 183, 444-451.

34. Expert Panel on Detection, Evaluation, and Treatment of High Blood Cholesterol in Adults (2001) Executive summary of the third report of the national cholesterol education program (NCEP) expert panel on detection, evaluation, and treatment of high blood cholesterol in adults (adult treatment panel III). JAMA 285, 2486-2497.

35. Levey AS, Stevens LA, Schmid CH et al. (2009) A new equation to estimate glomerular filtration rate. Ann Intern Med 150, 604-612.
36. Clifton PM (2011) Protein and coronary heart disease: the role of different protein sources. Curr Atheroscler Rep 13, 493-498.

37. Yahalom G, Kivity S, Segev S et al. (2014) Estimated glomerular filtration rate in a population with normal to mildly reduced renal function as predictor of cardiovascular disease. Eur J Prev Cardiol 21, 941-948.

38. Elmer PJ, Obarzanek E, Vollmer WM et al. (2006) Effects of comprehensive lifestyle modification on diet, weight, physical fitness, and blood pressure control: 18-month results of a randomized trial. Ann Intern Med 144, 485-495. 